\title{
Control of Isomerization in Ensembles of Nonrigid Molecules based on Classical and Quantum-mechanical Models, LiCN
}

\author{
Alexander A. Efimov \\ Department of Mathematics and Mechanics \\ St. Petersburg State University \\ 188194, St. Petersburg, Russia \\ aaefimov@mail.ru
}

Florentino Borondo

Departamento de Quimica, C-IX

Universidad Autonoma de Madrid

Cantoblanco-28049, Madrid, Spain

f.borondo@uam.es

\author{
Alexander L. Fradkov \\ Laboratory "Control of Complex Systems" \\ Inst. for Problems of Mechanical Engineeging RAS \\ 199178, St. Petersburg, Russia \\ fradkov@mail.ru
}

\author{
Mikhail S. Ananyevskiy \\ Department of Mathematics and Mechanics \\ St. Petersburg State University \\ 188194, St. Petersburg, Russia \\ msa@rusycon.ru
}

Rosa M. Benito

Departamento de Fisica, ETSI Agronomos

Universidad Politecnica de Madrid

28035 Madrid, Spain

rosamaria.benito@upm.es

\author{
Dmitry V. Yakubovich \\ Department of Mathematics \\ Universidad Autonoma de Madrid \\ Cantoblanco-28049, Madrid, Spain \\ dmitry.yakubovich@uam.es
}

Keywords - Nonlinear control, quantum control, isomerization, potential energy surface, intramolecular dynamics, Schrodinger equation, density matrix, LiCN.

Abstract-In this paper we report some investigations on the problem of controlling isomerization for small polyatomic non-rigid molecules, using the $\mathrm{LiNC} / \mathrm{LiCN}$ system as an example. Two methods of control in the classical ensemble of LiNC/LiCN system are described and analyzed by performing computer simulations for the corresponding canonical ensemble. The first method is based on controlling the total energy. The second one is based on changing the minimum energy path profile, and the potential energy surface for a certain "representative" configurations of the molecule. The algorithm used in both cases is based on the speed-gradient principle. The control function obtained in the classical mechanical study, with the total energy control algorithm, is subsequently applied to the quantum mechanical ensemble of $\mathrm{LiNC} / \mathrm{LiCN}$ molecules. The quantum mechanical calculations are carried out within a finite basis approximation, consisting of 14 energy levels and the corresponding eigenfunctions. A comparison between the simulation results for the classical and quantum models shows a reasonable similarity in the performance on the control.

\section{INTRODUCTION}

The control of molecular systems has attracted a growing interest since the beginning of the 1990's due to the development of femtosecond lasers, which constitute a practi-

This work was partially financially supported by Complex Program of the Presidium of RAS No 19 "Control of mechanical systems". The last author was supported by the Ramón and Cajal Programme (2002), the FEDER and the MEC Grant MTM2005-08350-C03-01 (Spain). cal tool for the realization of such control. Various methods, aimed at changing the natural course of a chemical reaction and creating new chemical technologies has been investigated by different authors (Brown and Rabitz, 2002; Butkovsky and Samoilenko, 1980).

It is well known that the topology of the potential energy surface (PES) contains essential information for the dynamics of the corresponding molecular system. The existence of several minima at the PES implies the existence of different stable configurations, having specific geometrical characteristics and chemical properties. At the thermodynamical equilibrium and in the absence of external fields, the percentage of molecules having any given configuration is determined by the depth of corresponding potential well. In this respect, it is interesting to consider the problem of changing at will the isomer concentration in the ensemble by applying an external electromagnetic (laser) field -i.e. the problem of controlling the isomerization reaction.

In the present work we apply some methods from the modern theory of nonlinear control to a model problem for the controlled isomerization of $\mathrm{LiNC} / \mathrm{LiCN}$, by presenting a synthesis with the study of the dynamics of the controlled ensemble. The LiNC/LiCN system has been extensively studied, specially in connection with the problem of quantum chaos (Borondo and Benito, 2005), as representative of a large class of molecules, and the corresponding dynamics thoroughly investigated (see (Benito at al, 1989), (Tarquis at al, 2001) and references therein). For example, these studies have explained why the fraction of less stable 
isomers $\mathrm{HCN}$ is greater than the fraction of more stable $\mathrm{HNC}$ in the atmosphere of some carbon stars (Harris at al, 1982).

The control algorithms, based on controlling the total energy of the system and on changing the form of a minimum energy path (MEP) and PES (the so called potential shaping) has been described elsewhere (Efimov et al, 2006). The performance of the algorithms is analyzed by computer modelling an ensemble consisting of 1000 molecules. Note that the thermal motion of the whole ensemble is complex and may be even chaotic, while the behavior of any single molecule is described by the Hamiltonian model. The control function is designed by means of a classical model and then used for the corresponding quantum mechanical ensemble of LiNC/LiCN molecules. Quantum mechanical calculations are performed in a finite-basis approximation consisting of 14 energy levels. The comparison of simulation results for classical and quantum mechanical models shows a reasonable agreement of the control performance in both cases.

This paper is the continuation of the paper (Efimov et al, 2006), where only the control of isomerization, based on classical model was considered.

\section{Classical Model for the Controlled System}

The molecular system $\mathrm{LiNC} / \mathrm{LiCN}$ is nonrigid with a very floppy motion in the angular coordinate, and its dynamics can be rather complex. At moderately small values of the energy, the system can pass between the two stable configurations corresponding to the isomers $\mathrm{LiCN}$ and LiNC, generating a chaotic dynamics. This is due to the possibility of relatively free motion of atom $\mathrm{Li}$ around the CN fragment, whose oscillations are practically uncoupled to the dynamics of the other molecular motions. Accordingly the dimensionality of the system can be reduced to two degrees of freedom (Benito at al, 1989), (Tarquis at al, 2001). The classical Hamiltonian of the system without control is given by

$H_{0}=\frac{P_{R}^{2}}{2 \mu_{L i-C N}}+\frac{1}{2}\left(\frac{1}{\mu_{L i-C N} R^{2}}+\frac{1}{\mu_{C-N} r_{e}^{2}}\right) P_{\theta}^{2}+V(R, \theta)$

where $R$ is the distance from the Li atom to the center of mass of the CN fragment, $\theta$ is the angle between the $\mathrm{N}-\mathrm{C}$ and $R$ vectors, and $P_{R}$ and $P_{\theta}$ are the corresponding conjugate momenta. Throughout the paper atomic units are used unless otherwise stated. The C-N distance is held frozen at its equilibrium value of $r_{e}=2.186$ The reduced masses are $\mu_{L i-C N}=10053$ and $\mu_{C-N}=11778$, respectively. The potential energy surface (PES) $V(R, \theta)$ is given by a 10 terms expansion in Legendre polynomials:

$$
V(R, \theta)=\sum_{k=0}^{9} V_{k}(R) P_{k}(\cos (\theta))
$$

fitted to quantum calculations and experimental data (Essers at al, 1982). As discussed in the Introduction, this PES has two potential wells corresponding to the stable linear isomers: $\mathrm{Li}-\mathrm{NC}\left(\theta=180^{\circ}, V=0\right)$ and the less sta- ble Li-CN $\left(\theta=0^{\circ}, V \approx 0.0104\right)$. They are separated by a barrier of approximately 0.0157 located at $\theta_{\text {saddle }} \approx 60.1^{\circ}$.

In order to control the dynamics of this molecular system we use an external electromagnetic (laser) field, which introduces a external force in the system dynamics. In this respect the molecule $\mathrm{LiNC} / \mathrm{LiCN}$ can be approximately considered as a dipole with unit charges, placed at the $\mathrm{Li}$ and center of mass of CN fragment, respectively. Thus, the full Hamiltonian of the controlled system is a sum of Hamiltonian $H_{0}$ with the dipole-external field interaction, $H_{1}$ :

$$
H_{1}=-q R E \cos (\theta-\phi) .
$$

Here $q=1$ is the dipole charge, $E$ is the intensity of external field, and $\phi$ is the angle between the vector of external field and the $\mathrm{N}-\mathrm{C}$ direction. Thus, the Hamiltonian of the molecular system LiNC/LiCN in an external electromagnetic field takes the form:

$$
\begin{gathered}
H=\frac{P_{R}^{2}}{2 \mu_{L i-C N}}+\frac{1}{2}\left(\frac{1}{\mu_{L i-C N} R^{2}}+\frac{1}{\mu_{C-N} r_{e}^{2}}\right) P_{\theta}^{2}+ \\
V(R, \theta)-R \cos (\theta-\phi) U
\end{gathered}
$$

where $U$ is the control function.

The substitution of (4) into the Hamilton equations:

$$
\begin{aligned}
\frac{d R}{d t} & =\frac{\partial H}{\partial P_{R}}, & \frac{d \theta}{d t} & =\frac{\partial H}{\partial P_{\theta}} \\
\frac{d P_{R}}{d t} & =-\frac{\partial H}{\partial R}, & \frac{d P_{\theta}}{d t} & =-\frac{\partial H}{\partial \theta}
\end{aligned}
$$

yields the equations of controlled molecular motion:

$$
\begin{gathered}
\dot{R}=\frac{1}{\mu_{L i-C N}} P_{R}, \dot{\theta}=\left(\frac{1}{\mu_{L i-C N} R^{2}}+\frac{1}{\mu_{C-N} r_{e}^{2}}\right) P_{\theta} \\
\dot{P}_{R}=\frac{P_{\theta}^{2}}{\mu_{L i-C N} R^{3}}-\frac{\partial V(R, \theta)}{\partial R}+\cos (\theta-\phi) U \\
\dot{P}_{\theta}=-\frac{\partial V(R, \theta)}{\partial \theta}-R \sin (\theta-\phi) U
\end{gathered}
$$

These equations of motion are essentially nonlinear, with the different degrees of freedom coupled both kinetically and potentially. The corresponding integration was carried out by adapting in MATLAB a 2-nd order central differences method. The time step used is 3.074 (200 times smaller than the corresponding characteristic vibrational symmetric stretch frequency in $\mathrm{LiCN}$ isomer.

\section{Synthesis of Isomerization Control}

The main goal of control for the molecular ensemble under consideration is to increase the fraction of less stable isomer LiCN present in it. One of the possible approaches to achieve control for the molecular ensemble consists in the realization of the following three stages:

1. A choice of an element of the ensemble, which adequately represents it (in a certain sense) - "the representative molecule".

2. The control of this "representative molecule". In our case this means the stabilization of the system fluctuations around the less stable configuration LiCN. 
3. The implementation of the synthesized control function for the molecular ensemble as a function of time by using impulses of duration $T_{1}$ through time intervals $T_{2}$.

The main idea is that if the control algorithm is robust enough, it should also work for molecules with configurations slightly different from that of the "representative molecule". Thus, as one of necessary stages of the isomerization control for molecular ensemble it is necessary to achieve control for one molecule.

In order to design the control of one molecule, we have to define the control goal. It is obvious that the $\mathrm{LiCN}$ molecule possesses appropriate dynamics, if the value of the variable $\theta$ is close to $0^{\circ}$ during the major part of time. For sufficiently high energies, an analytical determination of such situation is rather complicated because of possibility for molecules with close initial data to reveal different dynamics. But if the value of $\theta$ is variable and the system energy corresponds to the wanted potential well, the molecule stays in this well due to the lack of energy to overcome the potential barrier, and the molecule reveals the appropriate dynamics constantly. Thus we can define the control goal for one molecule as reaching of the potential well of LiCN minimum with system energy less than the energy of potential barrier, which will imply the stabilization of oscillations around the appropriate configuration.

\section{First Algorithm: Control of System Full Energy}

According to the model, the PES of our molecular system has two potential wells separated by an energy barrier. The goal of the control consists, actually, in moving the system to the LiCN potential well from that corresponding to LiNC, which is the deepest one. For this purpose it is necessary that the molecule cross the potential barrier. Thus, if initially the molecule is close to the bottom of the deepest potential well, it is necessary to increase its energy. However it is not enough, since in addition the stabilization of oscillations in the less deep potential well is also required. We then propose to decrease energy of the system after the representative molecule overcomes the isomerization barrier. We use the speed-gradient method to achieve this energy control.

Below, a brief description of the speed-gradient method is given to make the paper self-contained. More details can be found in (Fradkov at al, 1999). Let the controlled system be modelled as:

$$
\dot{x}=F(x, u)
$$

where $x \in R^{n}$ is the state and $u \in R^{m}$ is the input (control). Let the goal of control be expressed as the limit relation:

$$
Q(x(t)) \rightarrow 0 \text { when } t \rightarrow \infty
$$

In order to achieve the goal (11) we can apply the SGalgorithm in the finite form:

$$
u=-\Psi\left(\nabla_{u} \dot{Q}(x, u)\right)
$$

where $\dot{Q}=(\partial Q / \partial x) F(x, u)$ is the speed of change of $Q(x(t))$ along the trajectories of $(10)$, and the vector $\Psi(z)$ forms an acute angle with the vector $z$, i.e. $\Psi(z)^{T} z>0$ for $z \neq 0$ (the superscript "T" stands for transpose).

In our case, the control goal of control is either to increase or to decrease the system full energy (i.e. the Hamiltonian function $H_{0}$ ). Thus a possible control goal can be written as:

$$
Q=\left(H_{0}-H_{0}^{*}\right)^{2} \rightarrow 0, \text { when } t \rightarrow \infty
$$

where the constant $H_{0}^{*}$ is sufficiently large for energy increasing and sufficiently small for energy decreasing. After some algebra (using control in form (12)), we arrive at following control algorithm:

$$
U=-\gamma \nabla_{u}\left(\left(H_{0}-H_{0}^{*}\right) \dot{H}_{0}\right)
$$

In order to simplify this expression, we take into account that $\dot{H}_{0}=-\dot{H}_{U} U$, where $H_{U}=-R \cos (\theta-\phi)$ and eliminate energy (e.g. assuming that the system energy $H_{0}$ does not exceed the value $H_{0}^{*}$ in case of energy increasing, and conversely in case of energy decreasing) in (14), finally arriving to the following energy control algorithm:

$U=\left\{\begin{array}{l}U_{1}^{0} \cdot(\dot{R} \cos (\theta-\phi)-R \sin (\theta-\phi) \dot{\theta}), 0 \leq t \leq T_{1}^{*} \\ -U_{2}^{0} \cdot(\dot{R} \cos (\theta-\phi)-R \sin (\theta-\phi) \dot{\theta}), T_{1}^{*} \leq t \leq T_{1}\end{array}\right.$

where the algorithm parameters are the relative intensities, $U_{1}^{0}$ and $U_{2}^{0}$, for energy increasing and decreasing, and the total control time, $T_{1}$ (impulse duration), and time of control switching, $T_{1}^{*}$, (switching moment can be assigned initially or determined by some conditions).

\section{Second algorithm: Changing of a Minimum Energy Path Profile and Potential Energy Surface Form}

The basic idea of this approach consists in changing the PES form by means of the control, so that the new PES has only one stable minimum, corresponding to the $\mathrm{LiCN}$ isomer in our case. This can be done by introducing additional external forces into the system dynamics. If we additionally stabilize this new minimum by decreasing the oscillation energy, such combined actions will achieve the control goal, namely, the stabilization of the less stable configuration.

The modification of the system PES was carried out in such a way that the minimum energy path (MEP) $V_{\text {min }}(\theta)=\min _{R} V(R, \theta)$ for the modified PES is a monotonously increasing function of $\theta$ with a single minimum at $\theta=0$. This change is realized by substituting the appropriate control function in Hamiltonian (4), so that the new Hamiltonian potential energy function will be the sum of $V(R, \theta)$ and this new contribution. Note that if we add a function of $\theta$ to the potential function $V(R, \theta)$, then the new MEP will be the sum of old MEP and this function. Thus, setting a desired MEP profile satisfying this conditions, $V_{\min }^{\text {new }}(\theta)$, we obtain the following expression for the first component of control:

$$
U_{1}=\frac{V_{m i n}^{n e w}(\theta)-V_{\min }(\theta)}{H_{U}(R, \theta)}
$$

Moreover, we choose the new MEP $V_{\min }^{\text {new }}$ so that when $\theta=\theta_{\text {crit }}=(\phi+\pi / 2) \bmod \pi$ (i.e. when $\left.H_{U}(R, \theta)=0\right)$, the 
condition $V_{\text {min }}^{\text {new }}\left(\theta_{\text {crit }}\right)=V_{\text {min }}\left(\theta_{\text {crit }}\right)$ holds, in order to avoid singularities in the above expression. Thus, the value of the control can be determined as the ratio of corresponding derivatives when $\theta=\theta_{\text {crit }}$.

The stabilization of the single minimum of the new PES is achieved by decreasing the system energy in away analogous to the previous subsection. The combined control is the sum of the two components obtained. The intensity of energy decreasing and the total control time are the parameters of the algorithm.

\section{Isomerization Control for a Classical Ensemble of THE LiNC/LiCN Molecular SYSTEM}

As it was mentioned before, the main goal of control for the molecular ensemble under investigation is to increase the proportion of less stable isomers LiCN. The control function, obtained for some "representative molecule", is used to control the whole molecular ensemble. The precalculated control function is used to control the ensemble in an open-loop mode during a specified time interval $T_{1}$, so that during a time $T_{2}$ system remains uncontrolled, and then the process is repeated again. If the value of $T_{2}$ is sufficiently large and the system is not isolated, then the relaxation of system distribution to the equilibrium and recovering of macroparameters will take place, due to chaotic thermal mixing and the presence of the thermal exchange with the environment (thermostat). Thus, after each impulse approximately equal results will take place, if the reaction products are not separated or fixed. In such situation, the average proportion of $\mathrm{LiCN}$ isomer is the magnitude of interest. But if the reaction yield can be fixed, for example, when a chemical reaction with wanted isomer takes place, the situation changes. If the interval between impulses is so small that the distribution remains non-equilibrated, then a gradual increase of the proportion of the LiCN isomer can take place. In the present work we modelled only one impulse to demonstrate the potentialities of our approach.

Our simulation included a modelling of the initial equilibrium ensemble at a fixed temperature. These ensembles satisfy the Gibbs distribution:

$$
w(z)=C e^{-H(z) / k T}
$$

where $w(z)$ is the frequency distribution in the phase space, $k$ is the Boltzmann constant, $H(z)$ is the Hamiltonian (the full energy) of the system, and $C$ is a normalization constant. To model classical canonical Gibbs ensembles a method, based on producing microcanonical ensembles and selecting the molecules in compliance with relative probabilities of the corresponding state, was used. We modelled the initial canonical Gibbs ensemble of 1000 molecules at $300 K$.

For control we should choose a "representative molecule" of the ensemble; something that can be done by different methods. In our case we take as the main factor that a maximum of molecules have initial configurations close to the given one, or, rather, they have a close angle dynamics. The reason for that is that the algorithms applied are robust with respect to initial data and should act upon angle dynamics. Molecules with close angle dynamics are defined by the closeness both in energy and angle values.

It is natural to measure the efficiency of the isomerization control by the portion of the wanted isomer in the controlled ensemble. But, as it was shown above, the determination of appropriate dynamics for one molecule is rather complicated. If the molecule has sufficiently small energy (less than isomerization barrier), then we can assign it to one or another isomer, but for molecules with relatively high energies this often cannot be done. One of possible ways to estimate the control efficiency is to calculate the fraction of molecules with $\theta \leq \theta_{\text {crit }}$. This characteristic is appropriate in our case for the following reasons. In the first place, for ensembles with low energy, it adequately shows the fraction of wanted isomers - if molecule is in corresponding potential well, the condition $\theta \leq \theta_{\text {crit }}$ holds permanently. Second, for ensembles with relatively high energy, this value also shows adequately the proportion of LiCN isomer, because for molecules that can be considered as belonging to the wanted isomer, the condition $\theta \leq \theta_{\text {crit }}$ holds during most of the time, while the molecules, which walk from one configuration to another, very often shows an almost uniform angle distribution, and therefore distort the whole angle distribution rather weakly.

To estimate the control efficiency it is necessary to compare the results of isomerization control and of intensive equilibrium heating (up to the same mean energy). Only the excess of output of wanted isomer in controlled ensemble in comparison with that in the ensemble, heated to the same mean energy, can be treated as a true efficient control output. The procedures described were carried out to design isomerization control for initially canonical Gibbs ensemble at the room temperature $300 K$ with molecules, uniformly distributed with respect to the orientation angle $\phi \in[02 \pi]$. The control functions were precalculated for the selected "representative" molecule by applying both algorithms. In both cases the control was switched off when oscillations near $\theta=0$ with sufficiently small energy took place.

To control the "representative" molecule full energy, we used the algorithm given by (15) with $U_{1}^{0}=0.25, U_{2}^{0}=0.5$ The angle $\phi$ for the "representative" molecule was chosen to be equal $0.36 \pi$, which is slightly greater than $\theta_{\text {saddle }} \approx$ $0.33 \pi$.

In the second algorithm, to change the MEP profile of the "representative" molecule we chose the desired form of MEP as quadratic with the minimum in $\theta=0$. The coefficient of quadratic dependence was selected so that for $\theta=\theta_{\text {crit }}=\phi+\pi / 2$ the following condition holds: $V_{\text {min }}^{\text {new }}\left(\theta_{\text {crit }}\right)=V_{\text {min }}\left(\theta_{\text {crit }}\right)$. Thus the desired MEP is given by equation:

$$
V_{\text {min }}^{\text {new }}\left(\theta_{\text {crit }}\right)=\beta \theta_{\text {crit }}^{2}
$$

where

$$
\beta=\frac{V_{\text {min }}\left(\theta_{c r i t}\right)}{\theta_{c r i t}^{2}}
$$

The first component of control is given by the ratio:

$$
U_{1}=\frac{\beta \theta^{2}-V_{\min }(\theta)}{H_{U}(R, \theta)}
$$


The second component of control has the following form:

$$
U_{2}=-U_{0}(\dot{R} \cos (\theta-\phi)-R \sin (\theta-\phi) \dot{\theta}
$$

with $U_{0}=0.3$. The angle $\phi$ for the "representative" molecule was chosen to be equal $\pi / 4$, with the corresponding values $\theta_{\text {crit }}=3 \pi / 4$ and $\beta=0.0011$. The resulting control is a sum of these two components, at that first component is omitted when the system drops into appropriate potential well.

The results of control for classical ensemble are presented on the figures in the last page. Figures 1 and 3 show, respectively, the control functions obtained by the method of control of system full energy and by the method of changing of MEP profile. These control functions as functions of time are then used to control all molecules of the ensemble. Figures 2 and and 4 show the dynamics of the proportion of the $\mathrm{LiCN}$ isomer in controlled ensemble as functions of time (solid line) in comparison with the proportion of $\mathrm{LiCN}$ isomer in the equilibrium ensemble with the same mean energy (dashed line) (i.e. results of intensive equilibrium heating). The main conclusions are as follows: these two algorithms are rather effective: they are $1.5-2$ times more effective than the intensive equilibrium heating (for appropriate control parameters).

\section{QuAntum Model of CONTROLled System}

In this section the corresponding quantum-mechanical model for molecular ensemble of $\mathrm{LiCN}$ is considered:

$$
i \hbar \frac{\partial \rho}{\partial t}=\left[H_{0}, \rho\right]+E(t)\left[H_{1}, \rho\right]
$$

where $\rho$ is the density matrix, $E(t)$ is the intensity of external field (control), $H_{0}$ is the Hamiltonian operator for system without control, and $H_{1}$ is the potential energy operator of $\mathrm{LiCN}$ in an external electromagnetic field.

Operators $H_{0}$ and $H_{1}$ in coordinate representation are as follows:

$$
\begin{gathered}
H_{0}=-\frac{\hbar^{2}}{2 \mu_{L i-C N} R^{2}} \frac{\partial}{\partial R}\left(R^{2} \frac{\partial}{\partial R}\right)-\frac{\hbar^{2}}{2}\left(\frac{1}{\mu_{L i-C N} R^{2}}+\right. \\
\left.+\frac{1}{\mu_{L i-N C} r_{e}^{2}}\right) \frac{1}{\sin \theta} \frac{\partial}{\partial \theta}\left(\sin \theta \frac{\partial}{\partial \theta}\right)+V(R, \theta) \\
H_{1}=-R \cos (\theta-\phi)
\end{gathered}
$$

Here $\mu_{L i-C N}, \mu_{L i-N C}, r_{e}, R, \theta, V(R, \theta), \phi$ are the same as in the classical model.

Corresponding to a classical model the portion of the $\mathrm{LiCN}$ isomer in the quantum ensemble is defined as the average value of the observable $G$ : $\hat{G}(t)=\operatorname{tr}(G \rho(t))$, where operator $G$ is

$$
G(R, \theta)= \begin{cases}1, & |\theta| \leq 60.1^{\circ} \\ 0, & |\theta|>60.1^{\circ}\end{cases}
$$

As for the classical model, we use the Gibbs distribution for the initial value of $\rho$ :

$$
\rho_{0}=A \sum_{j} \exp \left(\frac{-\lambda_{j}}{k T}\right) \hat{L}_{j},
$$

here $A$ is the normalization factor, and $\hat{L}_{j}$ are integral operators with kernels $L_{j}=\Psi_{j}(R, \theta) \Psi_{j}^{*}\left(R^{\prime}, \theta^{\prime}\right)$, where $\lambda_{j}$ and $\Psi_{j}(\cdot, \cdot)$ are eigenvalues and corresponding eigenfunctions of $\mathrm{H}_{0}$.

For computer simulation, a finite basis approximation was taken, with eigenvalues and eigenfunctions of unperturbed Schrodinger operator $H_{0}$ evaluates numerically. Also a digitization for $\phi$ was applied. The same input control function and simulation time as for classical case were used. All parameters of the system were the same as in the classical case. As the observable $G$ is not an invariant of the unperturbed system, we expected complicated (may be chaotic) oscillations of its average value. The results of quantum simulation with MEP changing control are shown in Fig. 5. The average value of $G$ starts from zero. Rapid initial growth is a consequence of the tunnel effect. The control algorithm stabilized oscillations at range $[0.04,0.08]$. The comparison of simulation results for classical and quantum-mechanical models shows a reasonable similarity of controlled systems performance.

\section{Conclusion}

A problem of controlled isomerization of non-rigid molecules based on classical and quantum-mechanical models was considered. Two methods of isomerization control in the classical ensemble of $\mathrm{LiNC} / \mathrm{LiCN}$ system have been described and analyzed by computer simulation for classical canonical ensemble. The first method is based on the control of full energy. The second one is based on changing the minimum energy path profile and of configuration of the potential energy surface for a certain "representative" molecule. Both control algorithms are designed by using the speed-gradient principle. Then, the control function found for the classical model with algorithm based on control of total energy have been tested for the quantummechanical ensemble of LiNC/LiCN molecules. The quantum mechanical modelling was performed by finite basis approximation consisting of 14 energy levels and corresponding eigenfunctions. A nontrivial problem of choice of initial condition for quantum-mechanical modelling adequate of classical one is solved by choosing the Gibbs distribution with the same temperature for both cases. A comparison of simulation results for classical and quantum mechanical models shows a reasonable similarity of controlled systems performance.

The next step of the research will be quantum mechanical modelling of the algorithm of control of the total energy of the $\mathrm{LiNC} / \mathrm{LiCN}$ system.

\section{AUTHOR BIOGRAPHIES}

Alexander A. Efimov is a PhD student at the Department of Mathematics and Mechanics of St. Petersburg State University. Works at the Laboratory "Control of Complex Systems" in the Institute for Problems of Mechanical Engineeging of Russian Academy of Science. His email address is: aaefimov@mail.ru.

Mikhail S. Ananyevskiy is a PhD student at the Department of Mathematics and Mechanics of St. Petersburg State University. Works at the Laboratory "Control of Complex Systems" in the Institute for Problems of Mechanical Engineeging of Russian 
Academy of Science. His email address is: msa@rusycon.ru.

Florentino Borondo works at the Departamento de Quimica, C-IX Universidad Autonoma de Madrid. His email address is: f.borondo@uam.es.

Rosa M. Benito works at the Departamento de Fisica, ETSI Agronomos, Universidad Politecnica de Madrid. Her email address is: rbenito@fis.etsia.upm.es.

Alexander L. Fradkov is head of the Laboratory "Control of Complex Systems" in the Institute for Problems of Mechanical Engineeging of Russian Academy of Science. His email address is: fradkov@mail.ru.

Dmitry V. Yakubovich works at the Department of Mathematics of Universidad Autonoma de Madrid.

\section{REFERENCES}

Efimov A, F. Borondo, and R. M. Benito. 2006. Control of Isomerization in Classical Ensembles of Nonrigid Molecular Systems, LiCN. Proceedings of 2nd International Conference "Physics and control".

Brown E. and H. Rabitz. 2002. Some mathematical and algorithmic challenges in the control of quantum dynamics phenomena. $J$. Math. Chem., Vol. 31, No. 1, 17-63.

Butkovsky A. G. and Yu. I. Samoilenko. Control of Quantum Mechanical Processes. Dodrecht: Kluwer, 1980. (In Russian, 1984.)

Borondo F. and R. M. Benito. Quantum Chaos in Floppy Molecular Systems: The LiCN Molecule, in Nonlinear Dynamics and Fundamental Interacions., edited by F. Khanna and D. Matrasulov. Dodrecht: Springer, 2005.

Essers R., J. Tennyson and P. E. S. Wormer. 1982. An SCF potential energy surface for lithium cyanide. Chem. Phys. Lett., Vol. 89, No. 3, 223-227.

Harris G. J., Ya. V. Pavlenko, H. R. A. Jones and J. Tennyson. 2004. The identification of HCN and HNC in carbon stars: model atmospheres, synthetic spectra and fits to observations in the $2.7-4 \mu m$ region. Mon. Not. R. Astron. Soc.

Benito R. M., F. Borondo, J.-H. Kim, B. G. Sumpter and G. S. Ezra. 1989. Comparison of classical and quantum phase space structure of nonrigid molecules, LiCN. Chem. Phys. Lett., Vol. 161, No. $1,60-66$.

Tarquis A. M. J. C. Losada, R. M. Benito, F. Borondo. 2001. Multifractal analysis of tori destruction in a molecular Hamiltonian system. Phys. Rev. E., Vol. 65, 016213.

Fradkov A. L., I. V. Miroshnik and V. O. Nikiforov. 1999. Nonlinear and Adaptive Control of Complex Systems. Dordrecht: Kluwer Academic Publishers.

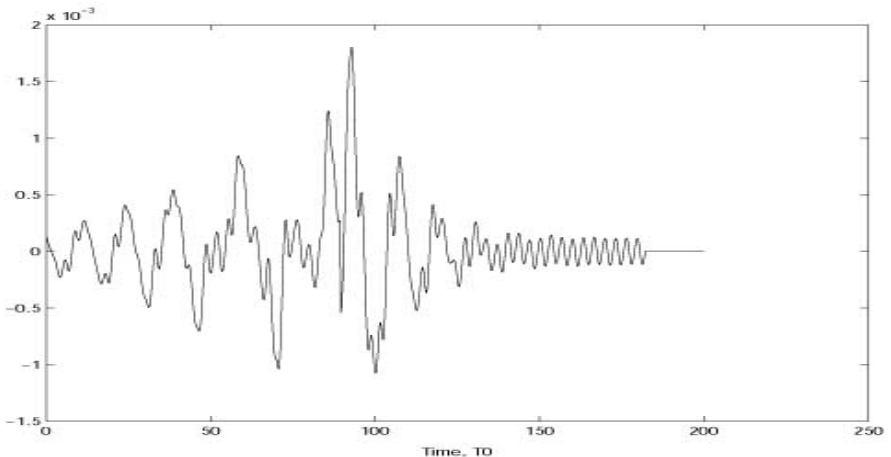

Fig. 1. Graph of the control function - full system energy control (after $T=180 T_{0}$ system remains uncontrolled).

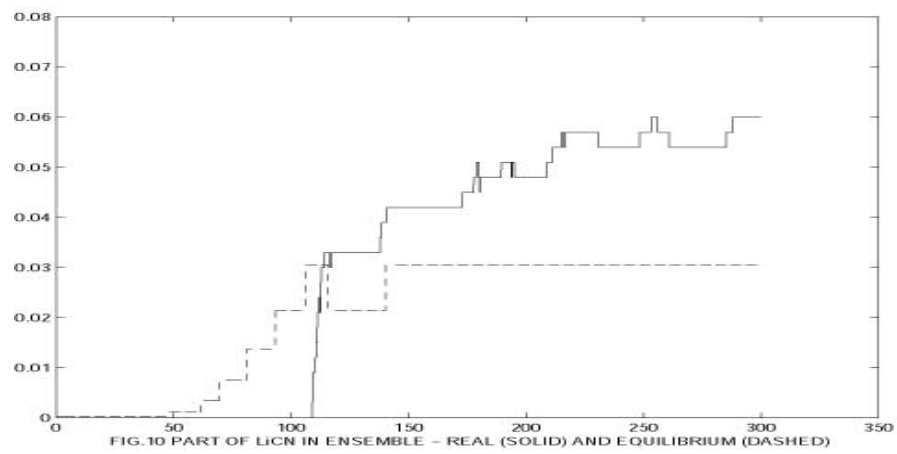

Fig. 2. Dynamics of the proportion of the LiCN isomer in the controlled ensemble. Solid line: results of full system energy control Dotted line: results of intensive heating (in the equilibrium ensemble with the same mean energy)

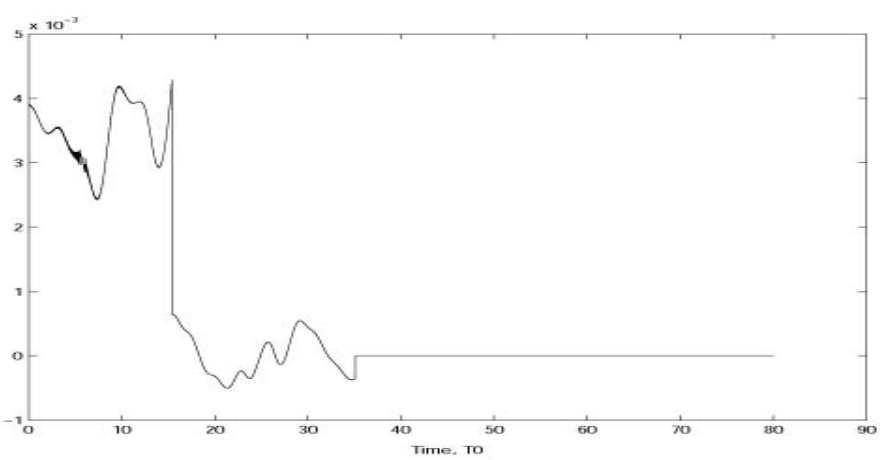

Fig. 3. Graph of the control function - MEP changing control (after $T=35 T_{0}$ system remains uncontrolled).

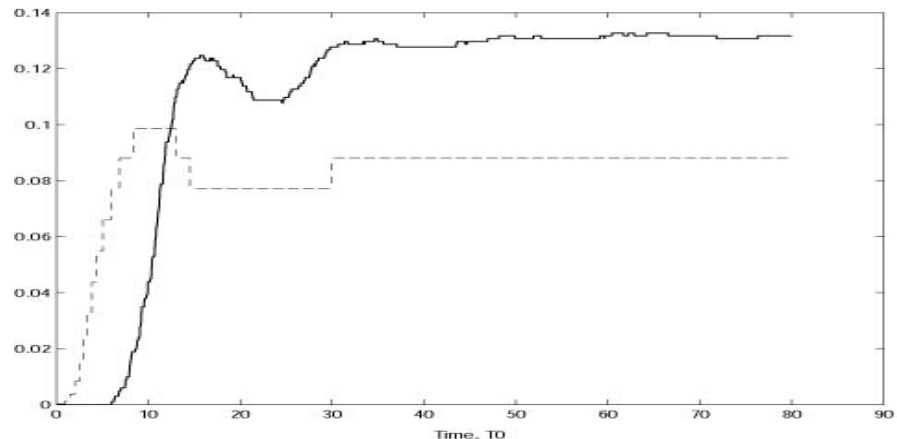

Fig. 4. Dynamics of the proportion of the $\mathrm{LiCN}$ isomer in the controlled ensemble. Solid line: results of the control based on changing the configuration of PES. Dotted line: results of intensive heating.

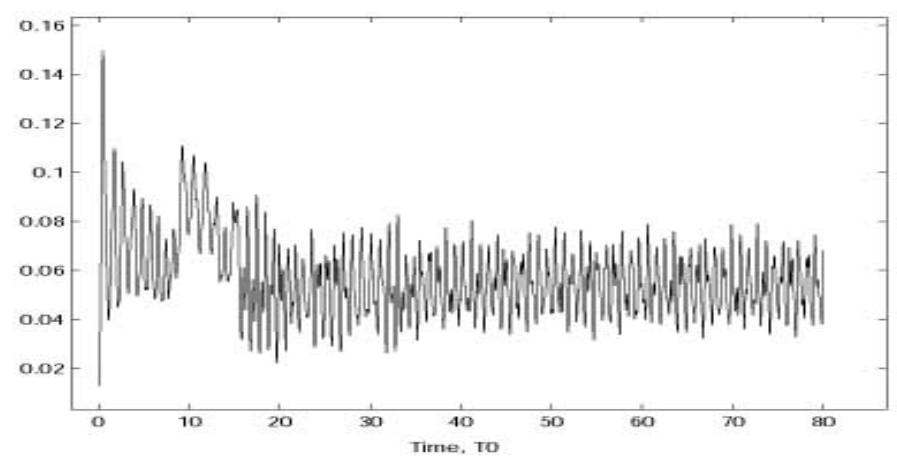

Fig. 5. Proportion of the $\mathrm{LiCN}$ isomer in the ensemble, quantum simulation. 